\title{
Etude De La Contamination Par Les Métaux Lourds Des Eaux Et Des Sédiments Au Voisinage De La Mine De Tighza (Maroc Central Oriental)
}

\author{
Farah El Hassani, PhD \\ Abdellah Boushaba, PES \\ Noureddine Raïs, PES \\ Université sidi Mohamed Ben Abdellah, Faculté des Sciences, Fès, Maroc \\ Lahcen Benaabidate, PES \\ Université sidi Mohamed Ben Abdellah, \\ Faculté des Sciences et Techniques, Fès, Maroc
}

doi: 10.19044/esj.2016.v12n6p158 URL:http://dx.doi.org/10.19044/esj.2016.v12n6p158

\begin{abstract}
The analyses performed on four stations upstream and eight downstream of the Tighzamine (Jebel Aouam) were carried out to assess the contamination degree from tailings discharges. From the standpoint of water and soil qualities, concentrations of trace and major elements (TME), including $\mathrm{Cd}, \mathrm{Mn}, \mathrm{Pb}$ and $\mathrm{Zn}$ which concentrations $\left(\mu \mathrm{gL}^{-1}\right)$ reach respectively 82, 49918, 669, 36858 in water samples and 443, 161, 23607, 19459 in sediments, exceed greatly, in the vicinity of the mine, the international standards. Depending on the TME analyzes, all downstream stations shows different degrees of contamination, whereas it is less or absent at the upstream stations. Downstream stations thus require water and soil treatment, since the contents TME are quite large and clearly present a danger not only in the surrounding environment for population, fauna and flora, but also over long distances especially as the waters of Tighza river flow into the oued Bouregreg and thus reach the Sidi Mohammed ben Abdellah Dam (Rabat) which is used as drinking water for urban consumption. It is therefore imperative to treat these waters, so that they become drinkable and avoid bad impacts to the population..
\end{abstract}

Keywords: Mine de Tighza, contamination, aquifère, métaux lourds

Résumé

Les analyses effectuées sur quatre stations en amont et huit en aval de la mine de Tighza (JbelAouam) sont faites pour approcher le degré de la 
contamination résultant des rejets de résidus miniers. Du point de vue qualité des eaux et des sols, les concentrations en éléments traces et majeurs (ETM), notamment le $\mathrm{Cd}, \mathrm{Mn}, \mathrm{Pb}$ et $\mathrm{Zn}$, dont les teneurs $\left(\mu \mathrm{gL}^{-1}\right.$ ) atteignent respectivement 82, 49918, 669 et 36858 dans les eaux et 443, 161, 23607 et 19459 dans les sédiments, dépassent largement, dans les alentours de la mine, les normes internationales admises pour les eaux douces naturelles. Tous les sites en aval de la mine présentent une contamination à des degrés différents, selon les ETM, alors que celle-ci est moindre ou inexistante en amont. Les stations en aval nécessitent donc un traitement des eaux et des sols puisque les teneurs en ETM sont assez importantes et présentent manifestement un danger aussi bien dans le milieu environnant pour la population, la faune et la flore, mais également sur de longues distances surtout que les eaux de l’oued Tighza se déversent dans l’oued Bou Regreg et donc atteignent le barrage Sidi Mohammed ben Abdellah; source d'alimentation en eau potable de la capitale Rabat. Il est donc impératif de traiter ces eaux pour qu'elles deviennent potables et éviter des impacts néfastes pour la population.

Mots-clés: Mine de Tighza, contamination, aquifère, métaux lourds

\section{Introduction}

L'étude de la contamination de l'environnement par les rejets contenant des minéraux lourds a été largement abordée par plusieurs auteurs. Baize (1977) et Akujobi (2012) ont mis en évidence que l'augmentation des concentrations des métaux lourds de contamination est générée essentiellement par l'activité anthropique. Certes, une activité minière, par les processus de l'extraction du minerai, son broyage généralement à l'air libre (poussières) et le rejet des résidus liquides et solides, entraine nécessairement une contamination du milieu environnant aussi bien par ses résidus, la poussière et l'eau non traitée qui se déverse dans la nature (Lee et al., 2001).

Les activités minières qu’a connues et connaît encore le Maroc depuis le début du siècle dernier constitue un point d'appui au développement économique du pays (Agard et al., 1958 ; Boushaba et Michard, 2008 ; Cheilletz, 1984) . De nos jours, dans un contexte qui tend à la fermeture d'un certain nombre de sites, en raison des conditions économiques, on constate que les mines et surtout les déchets laissés après extraction peuvent porter atteinte à l'environnement.

Cette étude porte sur la mine de jbel Aouam (appelée aussi mine ou district de Tighza) qui est située à $6 \mathrm{Km}$ au nord-ouest de Mrirt, petite agglomération située à mis distance entre les villes d'Azrou et de Khénifra (fig. 1), aux coordonnées $33^{\circ} 07^{\prime}$ à $33^{\circ} 11^{\prime} \mathrm{N}$ et $05^{\circ} 34^{\prime}$ à $05^{\circ} 43^{\prime} \mathrm{W}$. Les 
altitudes sont dominées par le Jbel Aouam (1497 m), le village de Tighza se situant en contrebas $(1100 \mathrm{~m})$ et les site de IghramAwassar $(1230 \mathrm{~m})$ et de Sidi Ahmed (1300m). Pour essayer de comprendre si cette mine présente un danger pour l'environnement, à travers des investigations de terrain et l'étude d'importantes archives,nous avons effectué un échantillonnage des liquides et solides autour du district sur deux années, puis des analyses chimiquesdes principaux éléments traces métalliques (ETM)pour en quantifier les impacts.

Cette mine (district) s'étend dans une zone de collines à matériel paléozoïque schisteux à massifs et filons granitiques et micro-granitiques (Bouabdelli, 1989 ; Cheilletz, 1984 ; Nerci, 2006).

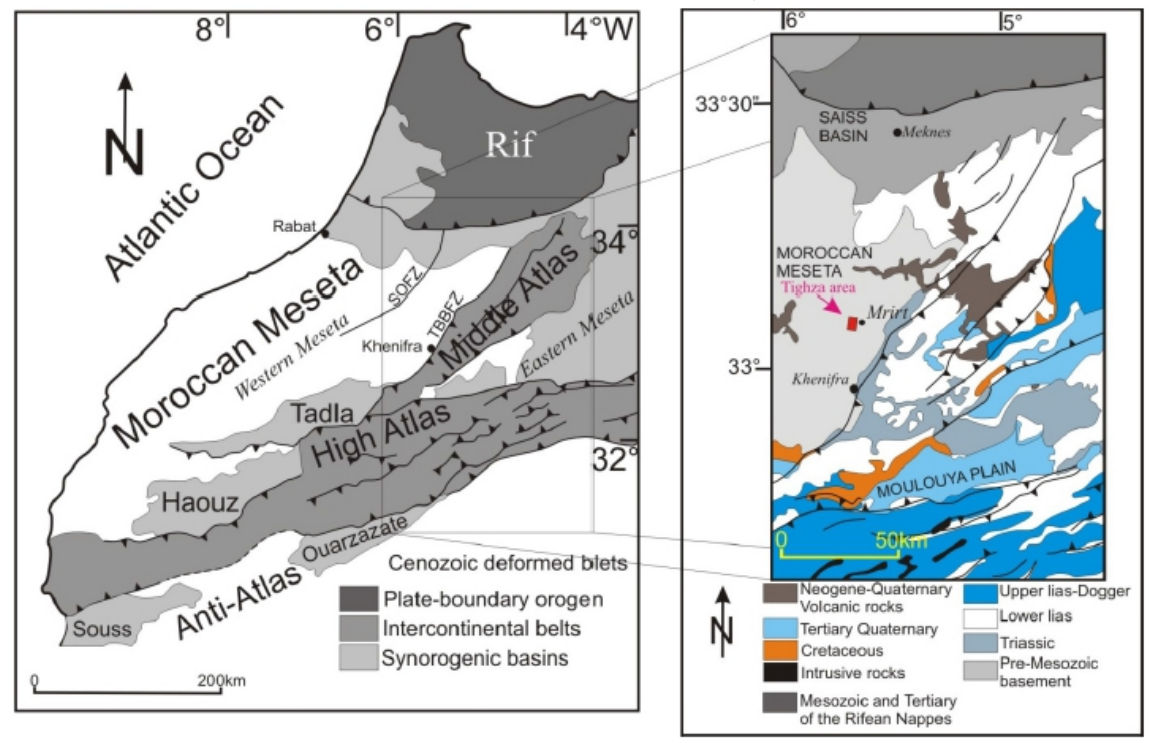

Figure 1 : Localisation de la zone d’étude (Eldurci, 2009)

\section{Matériels et Méthodes}

La compagne d'échantillonnage des eaux et des sols nus (rhizosphériques) aussi bien en amont de la mine (1 à 4) qu'en aval a été effectuée en deux périodes Mai 2014 et Avril 2015.

Les analyses des éléments traces dans les eaux et les sédiments ont été effectuées au Laboratoire de l'Office National des Hydrocarbures et des Mines à Rabatpar Spectrométrie d'Emission Atomique avec Plasma à Couplage inductif (de marque HoribaJobin Yvon, type Activa). C'est une technique qui est particulièrement très sensible puisque l'échantillon, mis en solution, est nébulisé dans un plasma d'Argon allant jusqu'à $8000^{\circ} \mathrm{C}$, ce qui permet alors une excitation très efficace des atomes. Les eaux ont été analysées directement par SEA-ICP sans préparation chimique, alors que les sédiments ont été pulvérisés à moins $70 \mu$ met ont été mis en solution par une 
attaque sur plaque chauffante en trois étapes : par le mélange $(\mathrm{HF}+\mathrm{HCl})$ puis par le mélange $\left(\mathrm{HNO}_{3}+\mathrm{HCl}\right)$ et enfin $\mathrm{HCl}$.

Les paramètres physicochimiques sont mesurés in situ grâce à un appareil multi-paramètres de type WTW.

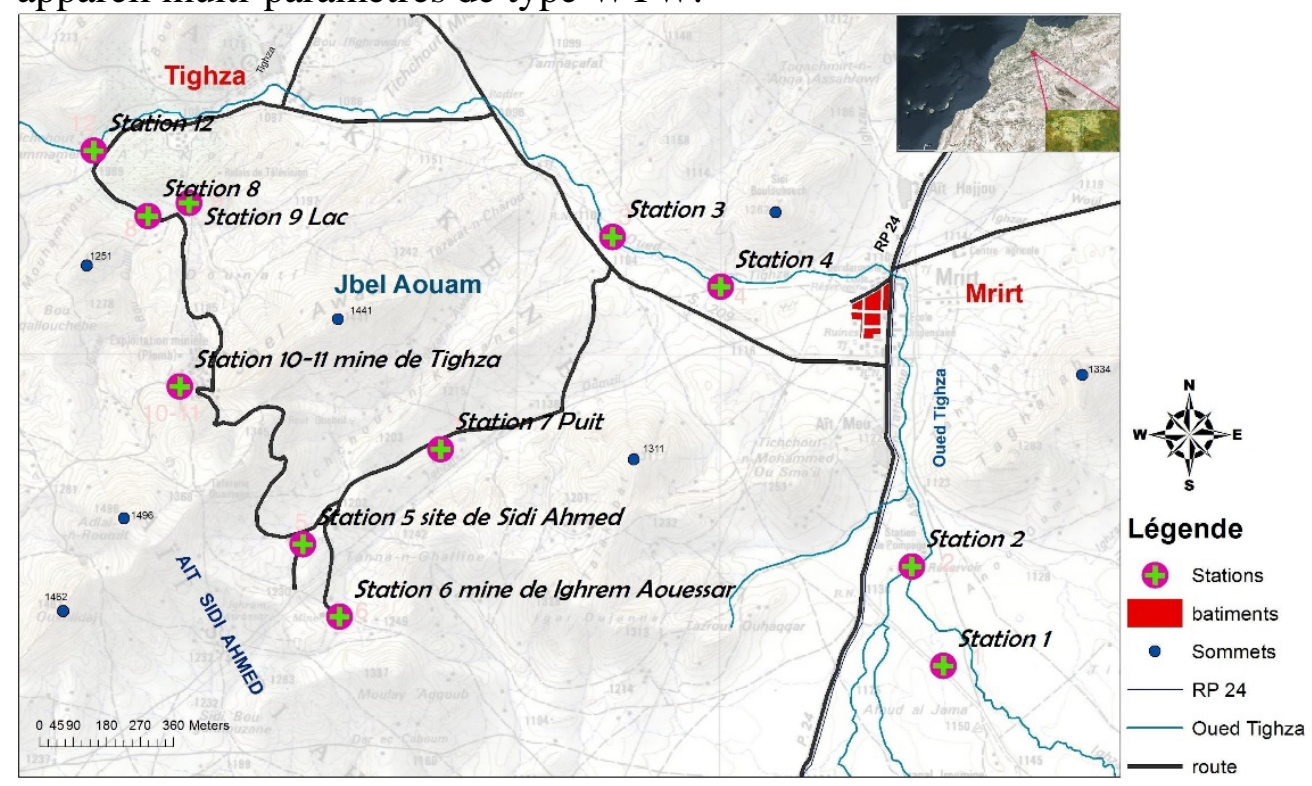

Figure 2 : Les sites d'échantillonnage ; 1 à 4 en amont de la mine; 5 à 12 en aval

\section{Résultats des analyses chimiques}

La présente étude concerne l'évaluation du degré de contaminationdes ressources hydriques par les minéraux lourds (Al, Cd, Co, $\mathrm{Cr}, \mathrm{Cu}, \mathrm{Mn}, \mathrm{Ni}, \mathrm{Pb}$ et $\mathrm{Zn}$ ). D’un autre côté, les métaux lourds (Ag, As, Cd, $\mathrm{Co}, \mathrm{Cr}, \mathrm{Cu}, \mathrm{Mn}, \mathrm{Pb}, \mathrm{V}$ et $\mathrm{Zn}$ ) ont été aussi analysés dans les sédiments qui se trouvent dans les cours d'eau de la région (fig.2) et ce dans le but d'identifier le degré de la contamination et sa variation au voisinage de la mine, aussi bien à son amont (stations 1 à 4) qu'à son aval (sites 5 à 12).

\section{Teneurs en métaux lourds dans les eaux et les sédiments de la campagne} Mai 2014

La répartition des teneurs en métaux lourds obtenus de l'analyse chimique des échantillons liquides pris aux alentours de la mine de Tighza est illustrée dans la figure 3. Cette figure montre bien que de la charpente des métaux lourds étudiés se dégagent cinq éléments principaux; Cadmium $(\mathrm{Cd})$, Cuivre $(\mathrm{Cu})$ et Arsenic (As), Manganèse $(\mathrm{Mn})$ et Zinc ( $\mathrm{Zn})$ qui affichent des teneurs largement supérieurs par rapport aux autres métaux lourds et qui dépassent $400 \mu \mathrm{gL}^{-1}$ et que les deux derniers éléments leurs concentrations dépassent $30.10^{3} \mu \mathrm{gL}^{-1}$. 

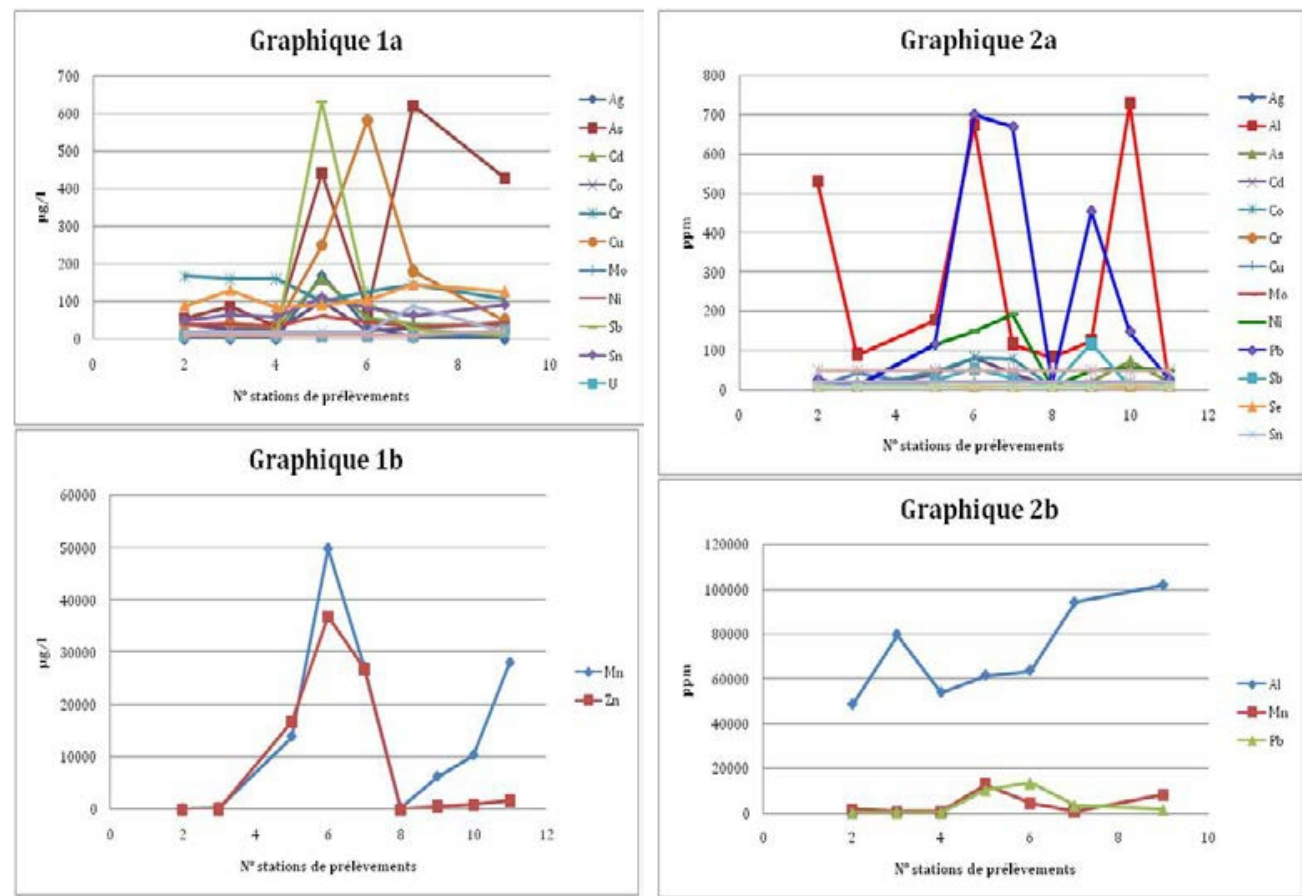

Figure 3 : Teneurs en métaux lourds dans les eaux (1) et les sédiments (2) de la campagne Mai 2014

Teneurs en métaux lourds dans les eaux et les sédiments de la campagne Avril 2015

En ce qui concerne la campagne d'Avril 2015, cinq éléments en trace se sont avérés avec des teneurs élevées supérieures à $200 \mu \mathrm{gL}^{-1}$ qui sont le Plomb $(\mathrm{Pb})$, l'Aluminium ( $\mathrm{Al})$, le Zinc $(\mathrm{Zn})$ et le Manganèse $(\mathrm{Mn})$ et que ces deux derniers éléments présentent des teneures qui restent excessives et dépassant $5.10^{3} \mu g L^{-1}$. 

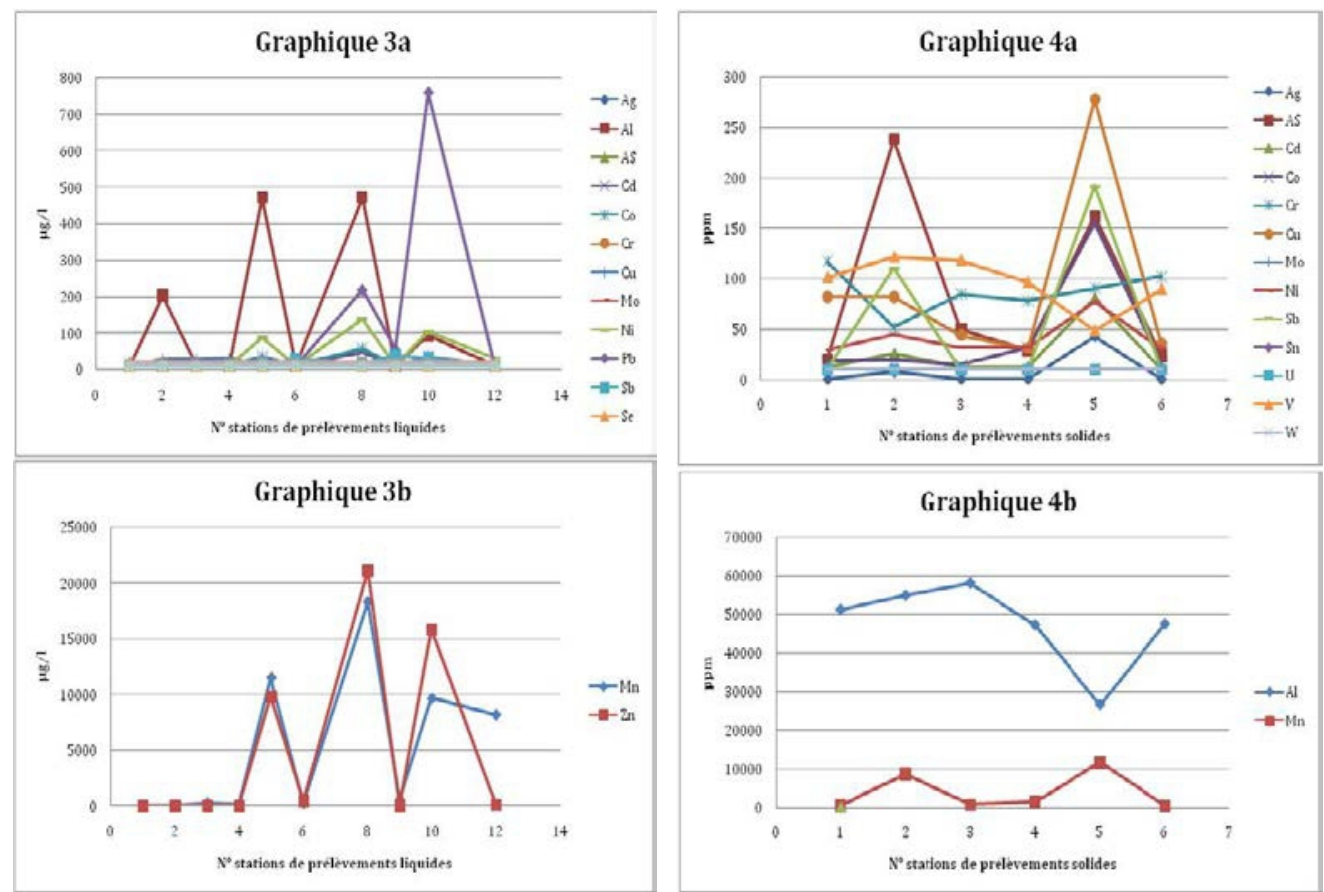

Figure 4 : Teneurs en métaux lourds dans les eaux (3) et les sédiments (4) de la campagne Avril 2015

\section{Discussion et interprétation des résultats}

$\mathrm{Au}$ Maroc, comme dans la plupart des pays en voie de développement, et en l'absence de législations sur la gestion des déchets miniers, une grande partie des minescontinuent à rejeter leurs déchets et eaux résiduels dans le milieu environnant.

\section{Les ressources hydriques}

Le diagnostic et la lecture des résultats des analyses chimiques des métaux lourds étudiés dans le district de Tizghaont permis de faire une évaluation de l'état de l'environnement dans la région étudiée.

Sur le plan qualitatif des ressources hydriques, l'étude a permis de constater que les concentrations en éléments traces métalliques (ETM) ; Cd, $\mathrm{Cu}, \mathrm{Pb}$ et $\mathrm{Zn}$, dépassent, pour la plupart des sites, largement les concentrations moyennes des eaux douces naturelles données par Bowen (1979) (Tab.1), alors que les éléments Cd, Zn et Mn, et pour les sites 5, 6 et 7 , situés en aval de la mine, les concentrations obtenues dépassent les normes marocains des eaux d'irrigation (Bulletin Officiel, 2002) et les normes de l'Organisation Mondiale de la Santé (Normes OMS in Burnol et al., 2006). Pour le $\mathrm{Mn}$, à l'exception du site $\mathrm{n}^{\circ} 2$, les autres sites ont affiché des teneurs largement supérieures à la norme d'irrigation fixée par la législation marocaine $\left(200 \mu g L^{-1}\right)$. 
Tab. 1 : Teneurs en $\mu \mathrm{gL}^{-1}$ en $\mathrm{Cd}, \mathrm{Mn}, \mathrm{Pb}$ et $\mathrm{Zn}$ dans les eaux

\begin{tabular}{|c|c|c|c|c|c|}
\hline Sites & Période & $\mathrm{Cd}$ & $\mathrm{Mn}$ & $\mathrm{Pb}$ & $\mathrm{Zn}$ \\
\hline \multirow[b]{2}{*}{1} & Mai 2014 & \multicolumn{4}{|c|}{ A sec } \\
\hline & Avril 2015 & $<10$ & $<10$ & $<10$ & $<10$ \\
\hline \multirow{2}{*}{2} & Mai 2014 & $<10$ & 171 & 27 & 14 \\
\hline & Avril 2015 & $<10$ & 33 & 14 & $<10$ \\
\hline \multirow[b]{2}{*}{3} & Mai 2014 & $<10$ & 337 & $<10$ & 69 \\
\hline & Avril 2015 & $<10$ & 267 & $<10$ & $<10$ \\
\hline \multirow[b]{2}{*}{4} & Mai 2014 & - & - & - & \\
\hline & Avril 2015 & $<10$ & 135 & $<10$ & $<10$ \\
\hline \multirow[b]{2}{*}{5} & Mai 2014 & 39 & 13927 & 116 & 16793 \\
\hline & Avril 2015 & 24 & 11506 & $<10$ & 9794 \\
\hline \multirow[b]{2}{*}{6} & Mai 2014 & 82 & 49918 & 700 & 36858 \\
\hline & Avril 2015 & $<10$ & 275 & $<10$ & 461 \\
\hline \multirow{2}{*}{7} & Mai 2014 & 42 & 27305 & 669 & 26860 \\
\hline & Avril 2015 & - & - & - & \\
\hline \multirow[b]{2}{*}{8} & Mai 2014 & $<10$ & 348 & 16 & 41 \\
\hline & Avril 2015 & 48 & 18307 & 218 & 21134 \\
\hline \multirow{2}{*}{9} & Mai 2014 & $<10$ & 6286 & 454 & 542 \\
\hline & Avril 2015 & $<10$ & 580 & 56 & 60 \\
\hline \multirow[b]{2}{*}{10} & Mai 2014 & $<10$ & 10412 & 148 & 837 \\
\hline & Avril 2015 & 30 & 9665 & 761 & 15831 \\
\hline \multirow[b]{2}{*}{11} & Mai 2014 & $<10$ & 28086 & 27 & 1690 \\
\hline & Avril 2015 & - & - & - & - \\
\hline \multicolumn{2}{|c|}{ Bowen, 1979} & 0.1 & 3 & 3 & 15 \\
\hline \multicolumn{2}{|c|}{ Normes OMS } & 3 & 50 & 10 & - \\
\hline \multicolumn{2}{|c|}{ Normes irrigation Maroc } & 10 & $10^{3}$ & $5.10^{3}$ & $2.10^{3}$ \\
\hline
\end{tabular}

Par ailleurs, la lecture des valeurs obtenues et des graphiques (1a, 1b, 3a et $3 b$ ) ci-dessus montre que toutes les stations en aval présentent une contamination à des degrés différents, selon les ETM, alors que celle-ci est moindre ou inexistante en amont (stations 1, 2 et 4 notamment). Pour ces dernières, l'eau valable selon les différentes normes pour l'irrigation, devrait être traitée si l'on veut l'utiliser en tant qu'eau potable. Pour les sites 5 à 12, un traitement est absolument nécessaire puisque les teneurs en $\mathrm{Cd}, \mathrm{Cu}, \mathrm{Mn}$, $\mathrm{Ni}, \mathrm{Pb}$ et $\mathrm{Zn}$ sont assez importantes et présentent un danger aussi dans le milieu environnant que pour la population, la faune et la flore. Les apports miniers en ETM (particulièrement As et $\mathrm{Pb}$ ) dans l'oued Tighza ainsi que dans les lacs et réservoirs environnants, rendent ces milieux insalubres. Par conséquent, la pollution émanant des trois sites miniers de Tighza constituent un risque pour les ressources hydriques au voisinage de la mine et que ce 
risque pourrait s’étendre plus loin en aval. Il est donc impératif de traiter ces eaux pour qu'elles deviennent potables.

\section{Les sédiments}

Les sols dans la zone minière de Tighza reçoivent après l'évacuation des eaux dans le milieu, l'essentiel du flux des dépôts en ETM. Par rapport à la pollution de l'air et de l'eau, la pollution des sols est peu visible et semble de ce fait attirer moins l'attention de la société.

Le pompage des eaux résiduaires de la mine provoque des contaminations, par leur transfert via des canalisations, pour se retrouver finalement rejetées dans le cours d’eau principal de la région.

Les valeurs obtenues de l'analyse des ETM dans les sédiments échantillonnés dans les bordures des cours d’eau enregistrent également de fortes teneurs en ces éléments pour toutes stations en aval, qui, d’après les normes de Bowen (1979) (Tab.2),montrent que les éléments As, Cd, Co, Cr, $\mathrm{Cu}, \mathrm{Pb}$ et $\mathrm{Zn}$ existent avec de fort taux dans les environs de la mine,aussi bien en aval qu'en amont, et ce grâce à la circulation des eaux pollués par les rejets de la mine et probablement aussi par le dépôt des poussières dégagée lors des travaux externes à la mine.Le lessivage de ces sédiments par des eaux peut contribuer à leur contamination avec des teneurs qui pourraient dépasser les normes marocaines pour l'irrigation et celles de l’OMS.

Tab. 2 : Teneurs en en $\mu \mathrm{gL}^{-1}$ de quelques ETM dans les sédiments

\begin{tabular}{|c|c|c|c|c|c|c|c|c|c|}
\hline Sites & Période & $\overline{\text { As }}$ & $\mathrm{Cd}$ & Co & $\mathrm{Cr}$ & $\mathrm{Cu}$ & $\mathrm{Mn}$ & $\mathrm{Pb}$ & $\mathrm{Zn}$ \\
\hline & Mai 2014 & \multicolumn{8}{|c|}{ Cours d'eau sec } \\
\hline \multirow[t]{2}{*}{1} & Avril 2015 & $<20$ & 12 & 19 & 117 & 82 & 586 & 67 & 112 \\
\hline & Mai 2014 & 45 & 36 & 40 & 168 & 38 & 1837 & 178 & 187 \\
\hline \multirow[t]{2}{*}{2} & Avril 2015 & 238 & 26 & 20 & 52 & 82 & 8756 & 4958 & 1619 \\
\hline & Mai 2014 & 87 & 36 & 26 & 163 & 45 & 788 & 286 & 633 \\
\hline \multirow[t]{2}{*}{3} & Avril 2015 & 50 & 13 & 15 & 85 & 44 & 691 & 570 & 526 \\
\hline & Mai 2014 & 33 & 28 & 23 & 161 & 34 & 765 & 151 & 184 \\
\hline \multirow[t]{2}{*}{4} & Avril 2015 & 29 & 13 & 32 & 78 & 31 & 1536 & 70 & 131 \\
\hline & Mai 2014 & 443 & 161 & 102 & 100 & 251 & 12718 & 10281 & 19459 \\
\hline \multirow[t]{2}{*}{5} & Avril 2015 & 162 & 81 & 155 & 91 & 278 & 11736 & 23607 & 24784 \\
\hline & Mai 2014 & 75 & 57 & 23 & 125 & 584 & 4465 & 13500 & 4481 \\
\hline 6 & Avril 2015 & 23 & 10 & 16 & 102 & 36 & 492 & 124 & 199 \\
\hline \multicolumn{2}{|c|}{ Bowen, 1979} & - & 6 & 0.35 & 8 & 70 & 30 & 35 & - \\
\hline
\end{tabular}

\section{Conclusion}

L’étude que nous avons menée dans le district de Tighza nous permis de mettre en évidence des impacts directs et des répercussions indésirables de l'exploitation minière sur le milieu environnant. La région présente un haut potentiel de pollution et des perturbations de l'esthétique (résidus miniers laissés au hasard sans traitement), nécessitantuneréhabilitation paysagère au fur et à mesure des exploitations. Ceci passe par la restauration 
du patrimoine naturel en intégrant y les trois sites miniers par leur historique, leur écologie. Ce milieu présente actuellement une dégradation physico chimique des eaux de surface et très probablement aussi de la nappe phréatique attestée par la présence de concentrations élevées en ETM (As, $\mathrm{Cd}, \mathrm{Cu}, \mathrm{Pb}, \mathrm{Zn}$ ). La dégradation de la qualité physico-chimique des eaux est démontrée particulièrement dans toutes les stations en aval de la mine ; ce qui oblige l'arrêt immédiat des lâchées d'eau dans l'oued Tighza dont le cours s’achemine, via l’oued Bou regreg, jusqu'au barrage de Rabat (pour l'eau potable).

La contamination des sols par les ETM est également démontrée (teneurs élevées par rapport aux valeurs normales des sols non contaminés), notamment dans les alentours immédiats des trois sites miniers Sidi Ahmed, IghramAwassar et JbelAouam. Dans les deux cas (eaux et sols) des mesures urgentes et un traitement adéquat doivent être pris pour la sauvegarde de l'environnement et pour la protection de la faune et de la flore, sans oublier bien sur la population non seulement autour de la mine mais également sur le parcours des oueds Tighza, Bou regreg et le barrage de rabat.

\section{References:}

Agard J., Balcon J.M., Morin Ph., Etude géologique et métallogénique de la région minéralisée du JbelAouam (Maroc central). Notes Mém. Serv. Géol. Maroc, 132, 126 p. 1958

Akujobi C.O., Odu N.N., Okorondu S.I., Bioaccumulation of lead by Bacillus speciesisolatedfrompigwaste. Journal of Research in Biology $\mathrm{N}^{\circ} 2$ pp: 83-89. 2012

Baize D., Teneurs totales en éléments traces métalliques dans les sols (France). Références et stratégies d'interprétation. Paris : Inra éditions. 1997. Bouabdelli M., Tectonique et sédimentation dans un bassin orogénique: le sillon viséen d'Azrou-Khénifra (Est du massif hercynien central du Maroc). Thèse Doct. Etat, Univ. Louis Pasteur, Strasbourg, 262 p. 1989.

Boushaba A., Michard A., Le district polymétallique de Tighza-JbelAouam (Massif central) In Nouveaux guides géologiques et miniers du Maroc, - Vol 9, Notes et Mémoires du Service Géologique, N564 pp : 261-269. 2008.

Bowen H.J.M., Environmentalchemistry of the elements, AcademicPress, New York,pp. 49-62. 1979

Bulletin Officiel, Normes marocaines de qualité des eaux destinées à l'irrigation. BO. N5062 du 5/12/2002. 2002.

Burnol A., Duro L., Grive M., Eléments traces métalliques. Guide méthodologique. Recommandations pour la modélisation des transferts des éléments traces métalliques dans les sols et les eaux souterraines. Rapport final. INERIS-DRC-06-66246/DESP-R01a. 119p. 2006. 
Cheilletz A., Contribution à la géologie du district polymétallique (W-MoCu-Pb-Zn-Ag) du JbelAouam, Maroc Central. Thèse Doct. Etat, I.N.P.L., C.R.P.G., E.N.S.G. Nancy, 250 pp. 1984.

Lee C.G., Chon H.T., Jung M.C., Heavy metal contamination in the vicinity of the Daduk $\mathrm{Au}-\mathrm{Ag}-\mathrm{Pb}-\mathrm{Zn}$ mine in Korea. AppliedGeochemistry $\mathrm{N}^{\circ} 16$ pp :1377-1386. 2001.

Nerci K., Les minéralisations aurifères du district polymétallique de Tighza (Maroc central) : un exemple de mise en place périgranitique tardihercynienne. Thèse de l'Univ Orléans, 240p. 2006. 